\title{
The use of glowing wood as a source of luminescent culture of fungus mycelium
}

\author{
Puzyr AP, Medvedeva SE and Bondar VS
}

Institute of Biophysics, Siberian Branch of Russian Academy of Science, Akademgorodok, 660036 Krasnoyarsk, Russia,

Puzyr AP, Medvedeva SE, Bondar VS 2016 - The use of glowing wood as a source of luminescent culture of fungus mycelium. Mycosphere 7(1), 1-17, Doi 10.5943/mycosphere/7/1/1

\begin{abstract}
In studies of fungal bioluminescence, not only fruiting bodies and spores of the fungus, but also samples of luminescent wood, leaf litter or soil may need to be used to derive pure mycelial culture. This study describes an approach to isolating the culture of luminescent fungal mycelium from samples of light-emitting wood found on Borneo Island in November-December 2013. A GelDoc XR Imaging System (Bio-Rad Laboratories, Inc., U.S.) was used for the first time to monitor luminescence and select luminous samples. This study shows that for successful isolation of the culture of luminescent mycelium out of the luminescent wood found in the forest, it is imperative to keep the samples moist (mycelium alive until there is water), while immediate and aseptic delivery of the samples to the laboratory is not a crucial condition (inner layers of wood is "sterile"). Investigation of the growth features of the isolated mycelium in various growing conditions revealed some peculiar properties of its luminescence in comparison with the known luminescent cultures of basidiomycetes. When grown on solid nutrient media, mycelium exhibits low growth rates, long-lasting luminescence (140 days or longer), and emergence and disappearance of local zones with high levels of light emission. Mycelium produced in submerged culture does not emit light, and this effect must be caused by the absence or a very low level of the luminescent reaction substrate in the biomass. The luminescence system isolated from mycelial biomass did not induce luminescent reaction in vitro upon the addition of NADPH (recording intensity is $60-100 \mathrm{URL} / \mathrm{sec}$ ). We found that enzymes of the luminescence systems isolated from mycelium pellets retained their activity and catalyzed luminescent reaction when a hot extract of the luminous fungus Armillaria sp. (IBSO 2360) was added (near $1900 \mathrm{URL} / \mathrm{sec}$ ). The same effect was obtained after addition of hot extracts from the fruiting bodies of nonluminous higher fungi Pholiota squarrosa, Cortinarius sp., Hypholoma capnoides and Chroogomphus rutilus (near 3500 $\mathrm{URL} / \mathrm{sec}$ ). The pure culture of luminescent mycelium has been registered in the Culture Collection of IBP SB RAS as IBSO 2371; now it can be used for various in vivo and in vitro studies, including identification of the fungus.
\end{abstract}

Key words - Bioluminescence - culture of luminous mycelia - kinetics of luminescent reaction light emitting wood - luminous fungus

\section{Introduction}

Studies of basidiomycete bioluminescence are usually prompted by finding their light emitting fruiting bodies in a night forest. Taxonomic positions of the luminous fungi are evaluated 
by classical phenotypic methods of classifying and identifying organisms and by phylogenetic analysis - a modern approach, which serves as an important tool for identification of fungal species in addition to morphological characteristics (Chew et al. 2015). Isolates extracted from fruiting bodies are maintained in collections and are used in laboratory studies of bioluminescence of fungal mycelium.

There are, however, a few exceptions, when studies address luminescence of mycelia of a luminous fungus on its natural wood substrate, in the absence of fruiting bodies. Deheyn and Latz (2007) reported that "Mycelia of luminous fungus were hand-collected between 20 January and 7 February 2005, at night, in the rainforest of Bocas del Toro, Panama." The authors reported bioluminescence characteristics of natural wood samples: the light was yellow, long-lasting and unaltered during the process of collection. Thus, it was not known whether the fruiting bodies of the fungus also emit light, and the fungus could not be definitely associated with a specific taxonomic group.

Sabharwal et al. (1983) reported the characteristics of light emission in vivo for different samples of the shining wood collected from different trees that became luminescent during the monsoon, from a village near Lonevala in Maharashtra (India). The authors described a bioluminescent fungal system from a wood sample. That study suggested that water is not only important for emission but has a function in the kinetics of the reaction. The authors intended to continue their study and investigate the culture conditions for the growth of the fungal system in order to understand mechanism of light emission. There are, however, no published results of their further study.

The historical review on luminescent wood in coal and ore mines was written by the Swiss researcher Brandl (2011). Light emitting wood was described more details in the studies published in the first half of the 19th century. J.F. Heller, Professor of the University of Vienna, was among the first who associated luminescence of rotting wood with the presence of mycelium of luminous mushrooms. Von Derschau et al. suggested the conclusion that luminescence of wood was associated with the presence of fungal mycelium - von Derschau's letter was published in 1823 by the German botanist T.F.L. Nees von Esenbeck (quote by Harvey 1952, 1957).

Light emission by leaf litter with no fruiting bodies of luminous fungi was described, too. Jaczewski (1933) wrote in his book that Tulasne in 1898 and Molisch in 1904 had observed luminescence of the thick leaf litter of different deciduous trees such as beech, maple, and oak. These leaves were covered and threaded with white web-like mycelium, without fruiting bodies. The researchers assumed that the mycelium might be associated with the genus Anthina Fr. or Himantia Fr. or represent a stage in the life cycle of Marasmius sp. An important fact is that Molisch observed the light emission by this mycelium in pure culture.

During our expeditions on Borneo Island in 2013, we found luminescent fruiting bodies of fungi on coconut palm stumps and shells of rotten coconuts. At the same time, we observed luminescence of wood and dead leaves in the absence of fruiting bodies, although we do not know why they were not there. We can only assume that the time necessary for fruiting bodies to emerge and develop might be so short that we were unable to detect them.

The purpose of this study was to derive pure culture of luminous fungal mycelium out of wood samples and to investigate mycelium luminescence in laboratory culture and the luminescence system isolated from it.

\section{Materials \& Methods}

The search for objects emitting light in the night forest took place in the vicinity of North Borneo Biostation (GPS coordinates are: 06 $57^{\prime} 46.00^{\prime \prime} \mathrm{N}$; 116 $49^{\prime} 46.50^{\prime \prime} \mathrm{E}$ [http://borneobiostation.com/biostation/]). Luminous wood was found between November 25 and December 5, 2013. The light was emitted by the trunk of the coconut palm lying on the ground in the plantation of oil palms. Samples of luminescent wood were cut out, placed into polyethylene bags and brought to the Institute of Biophysics (IBP) SB RAS. The wood samples were placed into a thermostatic chamber (TSO-1/80, SPU, Russia) or a climate chamber (WTH-E155, Daihan 
Scientific Co.Ltd., Korea). In the thermostatic chamber, humidity was not regulated and was determined by evaporation of water poured into polyethylene bags, at a temperature of $27^{\circ} \mathrm{C}$. Samples in the climate chamber were maintained at a temperature of $25^{\circ} \mathrm{C}$ and humidity of $99.8 \%$.

Luminescent zones of the samples were visualized by using a GelDoc XR Imaging System (Bio-Rad Laboratories, Inc., U.S.). Samples (wood, Petri dishes or flasks with mycelium) were placed into Universal Hood II. With daylight lamps on, we obtained visible light images of the samples; with daylight lamps off, we detected luminescent zones of the samples. The conditions for detecting the luminescent signal were as follows: the diaphragm open maximally, the time of signal accumulation - up to $300 \mathrm{sec}$. At a low luminescence level, the image was enhanced by using the Transform function of the software: 1 - gamma 4.00, low 0.0 or 2 - gamma 4.00, low 60021. In this study, bioluminescence was defined as a detectable light signal accumulated for up to $300 \mathrm{sec}$.

By using the GelDoc XR Imaging System, we chose wood samples with a high level of luminescence, rinsed them in water to remove soil particles, and used them for preparing pure culture. Mycelium was prepared by using conventional microbiological procedures. In the Purifier® Biological Safety Cabinet (Labconco®, U.S.), the surface of the samples was sterilized over the flame of the spirit lamp, the wood was cleaved with a sterile scalpel and placed in Petri dishes on five nutrient media of different compositions (containing $15 \mathrm{~g} / \mathrm{L}$ of agar-agar):

1) Malt extract agar (dextrose $-2 \mathrm{~g} / \mathrm{L}$, malt extract $20 \mathrm{~g} / \mathrm{L}$, peptone $-1 \mathrm{~g} / \mathrm{L}$ ) - (M agar);

2) Potato-dextrose agar (potato extract - $200 \mathrm{~g} / \mathrm{L}$, dextrose - $20 \mathrm{~g} / \mathrm{L}$ ) - (PD agar);

3) Yeast extract-malt extract agar (yeast extract $-3 \mathrm{~g} / \mathrm{L}$, malt extract $-3 \mathrm{~g} / \mathrm{L}$, dextrose -10 $\mathrm{g} / \mathrm{L}$, peptone - $5 \mathrm{~g} / \mathrm{L}$ ) - (YM agar);

4) Yeast extract-cane sugar agar (yeast extract - $10 \mathrm{~g} / \mathrm{L}$, cane sugar - $100 \mathrm{~g} / \mathrm{L}$ ) - (YS agar);

5) Sabouraud agar (mycological peptone - $10 \mathrm{~g} / \mathrm{L}$, dextrose - $40 \mathrm{~g} / \mathrm{L}$ ) - (S agar).

Ready-to-use nutrient media (PD- and S-agar) or their ingredients were purchased from HiMedia Lab (India).

We also used two types of the solid nutrient medium $S$ agar with water replaced by wheat grain extract $(S+G)$ or extract of wood from which mycelium had been collected $(S+W)$.

Luminescent mycelium that had grown in Petri dishes and registered in the IBP Culture Collection (CCIBSO 836) as IBSO 2371 was used as inoculum for the culture in liquid nutrient medium. Mycelial biomass was separated from the substrate, rubbed through a metal sieve (mesh size of $2 \times 2 \mathrm{~mm}$ ), and placed into a 300-ml flask that contained $100 \mathrm{ml}$ PD medium. Cultivation was conducted on a Max Q 4000 incubating shaker (Thermo Scientific, U.S.) at a temperature of $27-29^{\circ} \mathrm{C}$ and constant agitation at $140 \mathrm{rpm}$. The pellets that had grown in the liquid nutrient medium were used to study luminescence of the fungal mycelium as the whole organism and for isolating the luminescence system.

To isolate the luminescence system, mycelial biomass was placed in the cooled $0.1 \mathrm{M}$ phosphate buffer solution ( $\mathrm{pH}$ 7.0) that contained 1\% BSA and homogenized ultrasonically (with a Volna UZTA system 0.63/22-0, Russia), using an ice bath. The homogenate was centrifuged at a temperature of $4^{\circ} \mathrm{C}$, at $40000 \mathrm{~g}$ for $30 \mathrm{~min}$ (Avanti ${ }^{\circledR} \mathrm{J}-\mathrm{E}$ centrifuge, Beckman-Coulter, U.S.). The sediment was discarded, and the supernatant (cold extract), containing components of the luminescence system, was frozen at a temperature of $-80^{\circ} \mathrm{C}\left(-86^{\circ} \mathrm{C}\right.$ Ultralow Freezer, NuAire, Inc., U.S.), freeze dried (in an LS-500 freeze drier, Russia), and stored at a temperature of $-20^{\circ} \mathrm{C}$ until use. For luminescence measurements, the lyophilic powder was dissolved in deionized water, which had been prepared in a Milli-Q system (Millipore, U.S.).

The reaction mixture for measuring activity of the isolated luminescence system was prepared by sequentially pouring $100 \mu \mathrm{l}$ cold extract and dropping $5 \mu \mathrm{l} 10 \mathrm{mM}$ NADPH into MCT200-C microtubes (Axygen Scientific, Inc., U.S.); then the mixture was supplemented with $5 \mu$ hot extract or solution of the substance tested. The magnitude and dynamics of the change in the light signal were monitored by using a Glomax ${ }^{\circledR}$ 20/20 luminometer (Promega BioSystems Sunnyvale, Inc., U.S.).

As the hot extract we used: (i) the cold extract of the biomass of the IBSO 2371 mycelium heated at $100^{\circ} \mathrm{C}$; (ii) hot aqueous extracts of the fruiting bodies of nonluminous fungi Pholiota 
squarrosa, Hypholoma capnoides, Cortinarius sp. and Chroogomphus rutilus, collected in the forest in the vicinity of Krasnoyarsk. Hot extracts were prepared as follows.

(i) An aliquot of the cold extract of mycelium IBSO $2371(100-150 \mu \mathrm{l})$ prepared by dissolution of the lyophilic powder in water was heated in a boiling water bath for 2 min and then cooled on ice.

(ii) Fruiting bodies of the nonluminous fungi listed above were collected in the forest and rinsed in distilled water to remove foreign matter. The fruiting bodies were crushed to enhance extraction and transferred into a heat resistant glass. Deionized water was added to the biomass (1:4 water : biomass, v/v), and the sample was placed into the microwave oven (model MW 712BR, Samsung, Malaysia) and heated at $800 \mathrm{~W}$ until it boiled. Then, the sample was cooled on ice and centrifuged at $20000 \mathrm{~g}$ for $30 \mathrm{~min}$ at a temperature of $4^{\circ} \mathrm{C}$ (Avanti ${ }^{\circledR} \mathrm{J}$-E centrifuge). The sediment was discarded, and the supernatant was frozen at a temperature of $-80 \mathrm{oC}(-86 \mathrm{oC}$ Ultralow Freezer), freeze dried (in an LS-500 freeze drier), and stored at a temperature of $-20^{\circ} \mathrm{C}$ until use.

In the luminescence study, mycelial pellets were placed in the MCT-200-C microtubes, which were then installed in the Glomax ${ }^{\circledR}$ 20/20 luminometer. The levels of mycelial luminescence before and after the addition of solutions of different substances were monitored at a rate of 1 measurement per sec. Light emission intensity was expressed as relative light units (RLU) per sec.

Illustrations (the appearance and luminescence of wood and mycelium samples) were prepared using a GelDoc XR Imaging System (Bio-Rad Laboratories, Inc., U.S.) and a Canon EOS 6D camera. Photography in the light was done in the mode of ISO 200-500, $0.4-0.8 \mathrm{sec}$ exposure. Photography in the dark was done in the mode of ISO 1600-4000, 45 - $90 \mathrm{sec}$ exposure.

\section{Results}

\section{Light emitting wood}

Samples of luminescent wood that emitted greenish light were delivered to the laboratory in 3 days after sample collection, and the luminescence signal was recorded using the GelDoc XR Imaging System (Fig. 1).
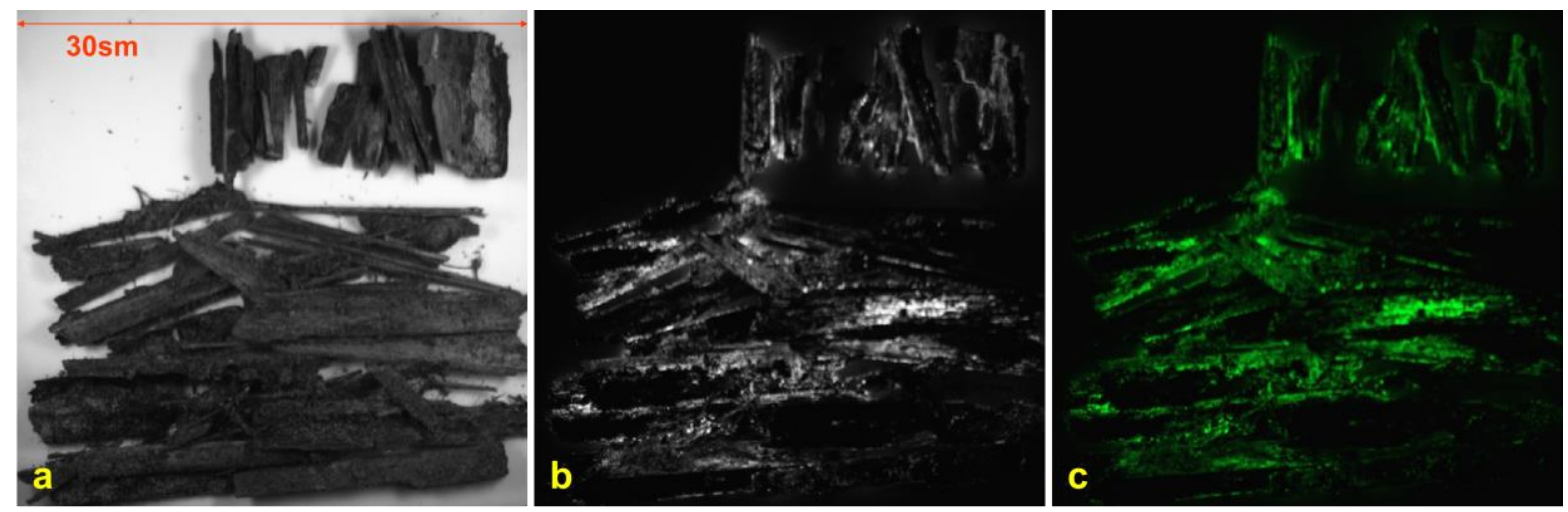

Fig. 1 - External appearance and luminescence of wood samples. a - the sample in the daylight; b luminescence of the sample in the dark; $\mathrm{c}$ - luminescence close to the natural spectral maximum of light emission by the sample (greenish light). The images are recorded by the GelDoc XR Imaging System with the SDC black-and-white camera: Recording mode: the time of accumulation $300 \mathrm{sec}$, transform function 1 .

After being maintained in humid conditions for extended periods of time, the wood samples remained luminescent. Aerial hyphae of mycelium, which were also luminescent, emerged on their surface. The mycelium of the study fungus typically has luminescent hyphae without any additional formations (Fig. 2). Mycelial hyphae with luminous spherical swellings were not typical (Figs. 3 and 4), and we detected them in 6 months after the wood samples were delivered to the laboratory. The spherical swellings on the hyphae had been observed for $1-1.5$ months, but then they 


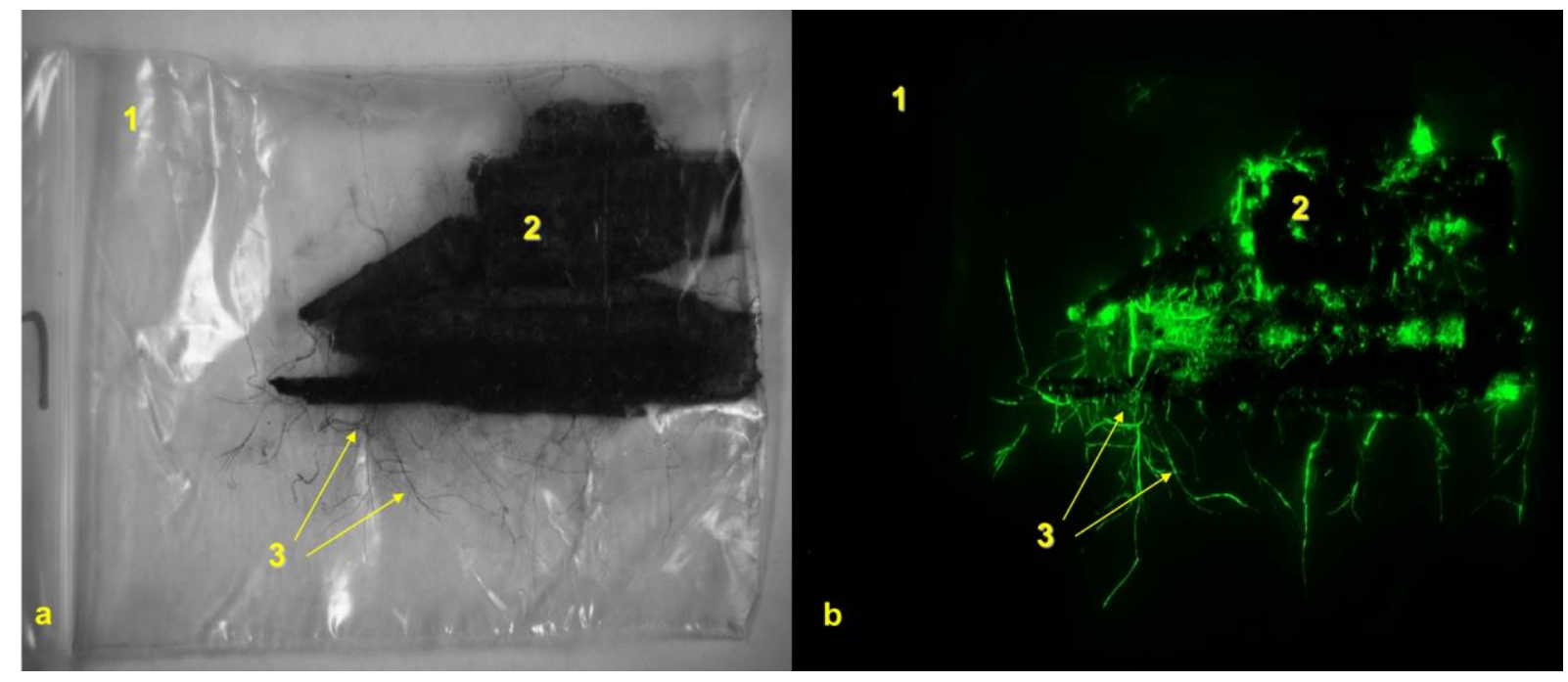

Fig. 2 - External appearance and luminescence of hyphae on the wood surface and aerial hyphae that had grown during storage of the wood sample under high humidity conditions, recorded by the GelDoc XR Imaging System: $a$ - the image of hyphae in the daylight; $b$ - luminescence of the hyphae. 1 - polyethylene bag, 2 - sample of wood, 3 - aerial hyphae on wood. Recording mode: the time of accumulation $300 \mathrm{sec}$, transform function 2. Wood samples were collected on December 5, 2013, imaging was performed on November 5, 2014.
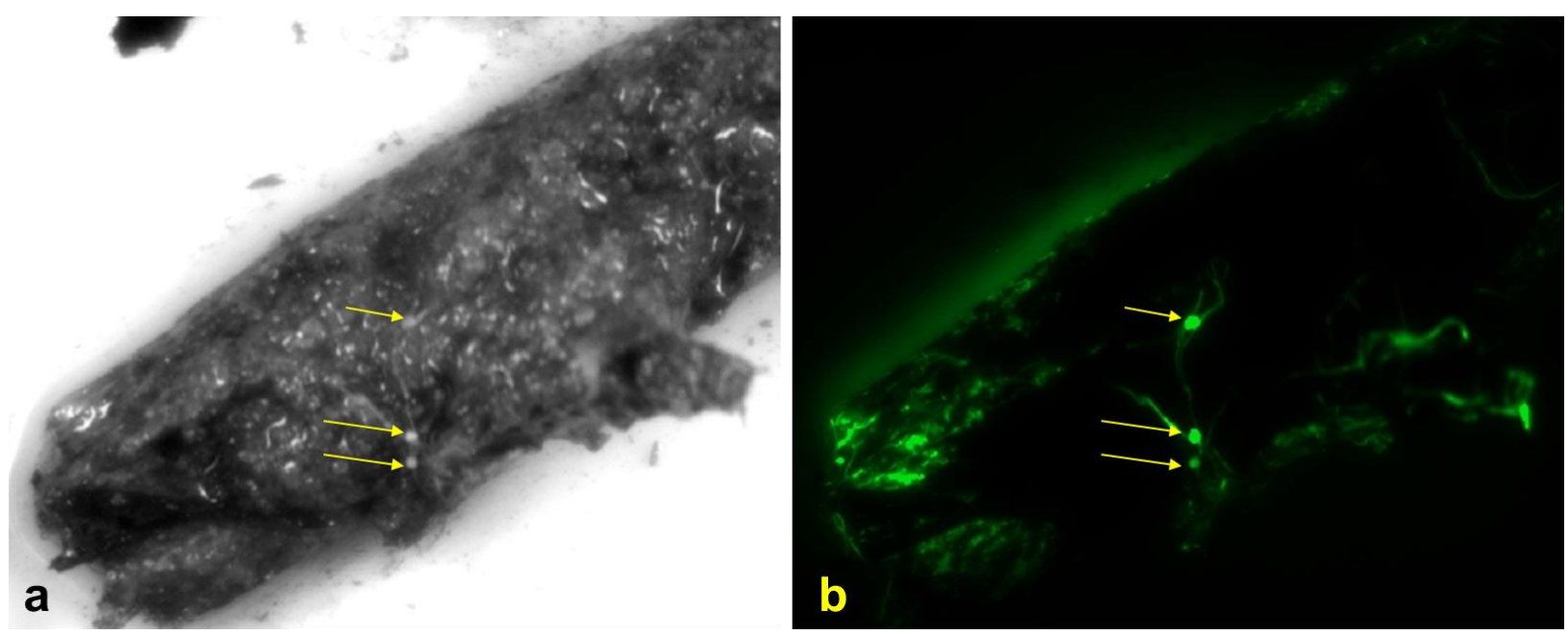

Fig. 3 - External appearance and luminescence of aerial hyphae with spherical swellings (shown by arrows) that had grown during storage of the wood sample under high humidity conditions, recorded by the GelDoc XR Imaging System: a - the image of hyphae with spherical swellings in the daylight; $b$ - luminescence of the hyphae. Recording mode: the time of accumulation 300 sec.

disappeared. As the hyphae and spherical swellings showed a high level of luminescence, we managed to record it by using the GelDoc XR Imaging System (Fig. 3) and the Canon EOS 6D camera (Fig. 4). Even now (after 22 months), new aerial hyphae, without any spherical swellings, are growing on the wood samples and emitting light.

\section{Preparation of pure culture of luminescent mycelium}

The image of wood samples (Fig. 1) shows that luminescence zones were not distributed uniformly. Imaging of luminescence by using the GelDoc XR Imaging System at a high magnification can precisely localize luminescent zones (Fig. 5). Based on the analysis of these images, we selected samples of luminescent wood for inoculation on solid nutrient media. 


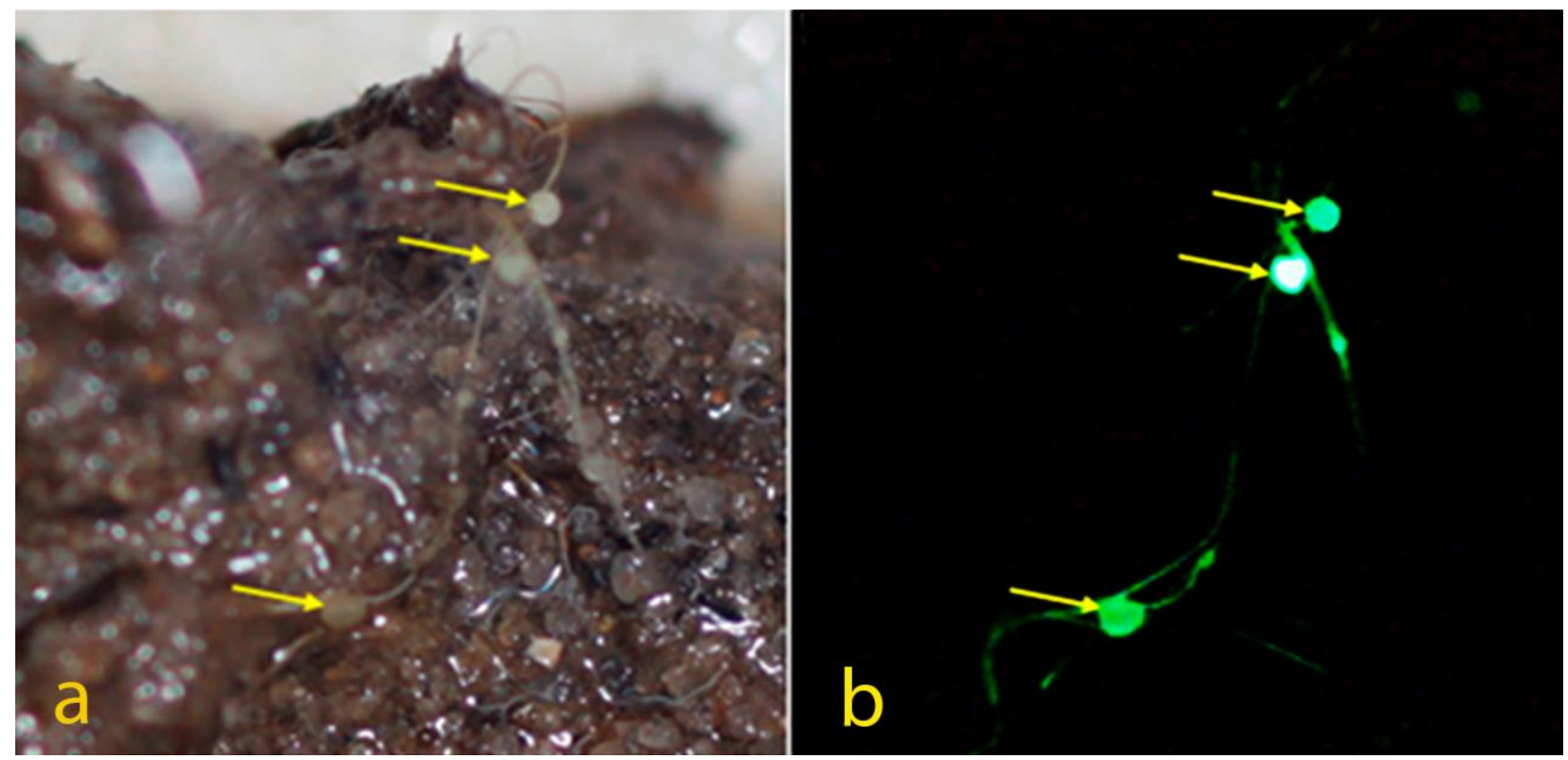

Fig. 4 - External appearance (a) and luminescence (b) of aerial hyphae with spherical swellings (shown by arrows) that had grown during storage of the wood sample under high humidity conditions, recorded by the Canon EOS 6D camera. Recording mode: AE, ISO 400, exposure 0.8 sec, daylight (a); AE, ISO 4000, exposure $45 \mathrm{sec}$, in the dark (b).
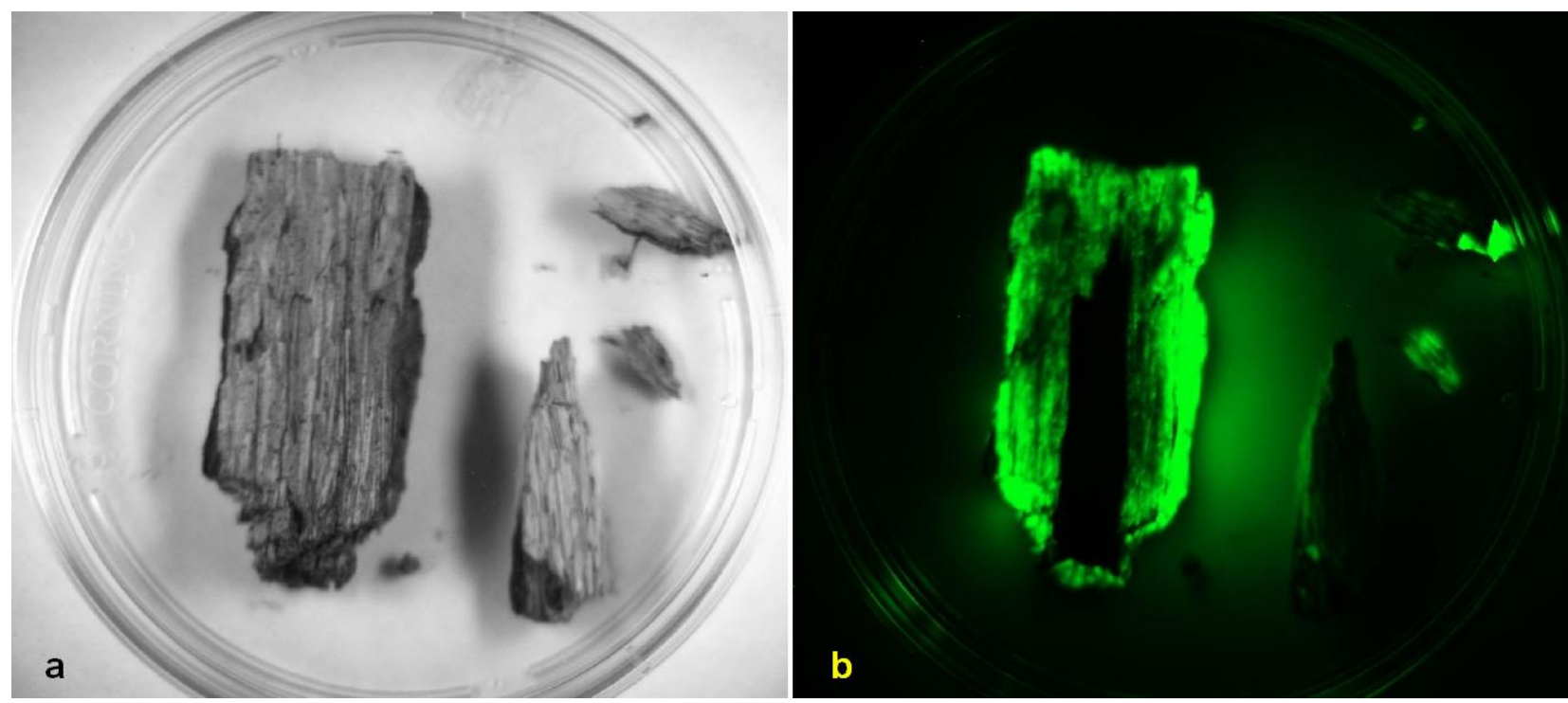

Fig. 5 - External appearance and luminescence of the wood sample selected for deriving the inoculum, recorded by the GelDoc XR Imaging System: a - the image of the sample in the daylight; $b$ - luminescence of the sample. Recording mode: the time of accumulation $300 \mathrm{sec}$, transform function 1 .

\section{Light emission of mycelium culture on solid nutrient medium}

Having compared the images obtained in the visible light and in the dark, we classified and selected brightly luminescent pieces of wood, placed them on the nutrient medium (Fig. 6), and monitored mycelium growth and luminescence on different media (Fig. 7).

The use of two techniques for recording luminescence (with a GelDoc XR Imaging System and Glomax ${ }^{\circledR}$ 20/20) enables better understanding of the material examined in this study. The luminometer, Glomax ${ }^{\circledR} 20 / 20$, provides integrated quantification of the luminescence signal, while the imaging system determines the localization and area of luminescence. Fig. 8 shows images of mycelium grown on nutrient media of different compositions for different periods of time. 


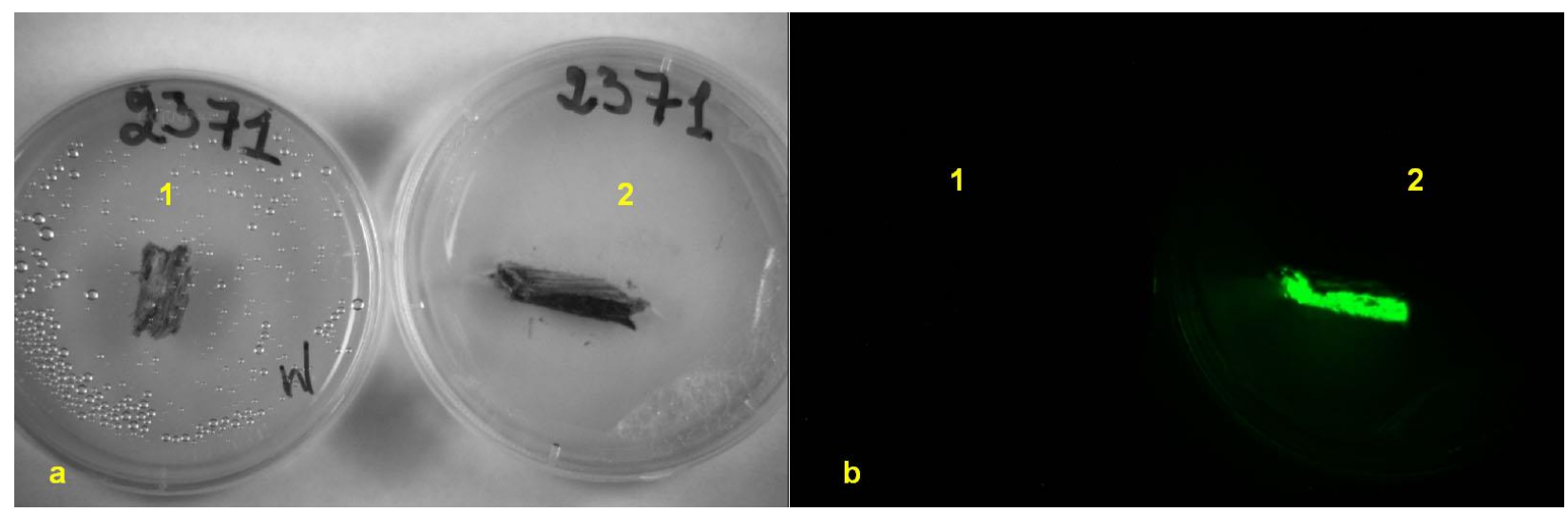

Fig. 6 - External appearance and luminescence of wood samples placed on nutrient medium $\mathrm{M}$ on 60-mm-diameter Petri dishes, recorded by the GelDoc XR Imaging System: a - the image of the samples in the daylight; $b$ - luminescence of the samples. Petri dishes: 1 - with non-luminescent sample of wood, 2 - with luminescent sample of wood. Recording mode: the time of accumulation $300 \mathrm{sec}$, transform function 2.

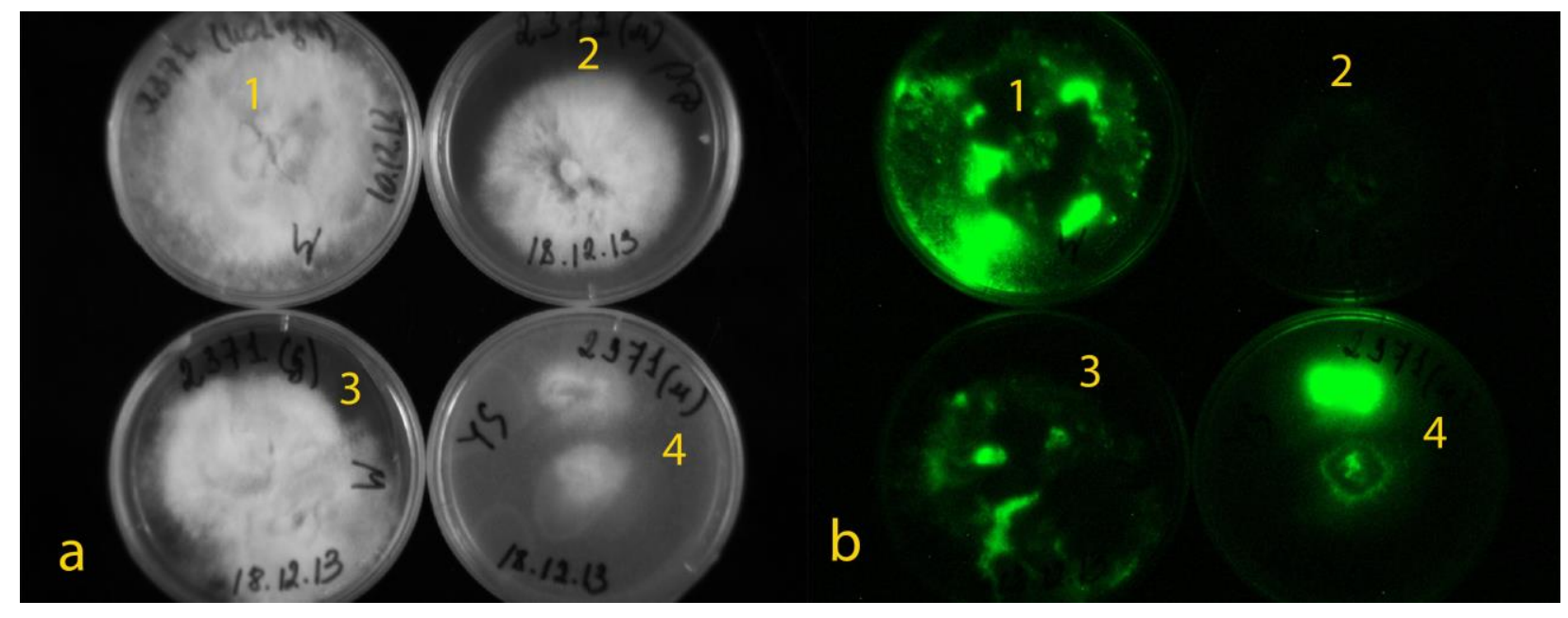

Fig. 7 - External appearance and luminescence of mycelium after cultivation of the fungus on different nutrient media in Petri dishes. Petri dish 1 - nutrient medium M, cultivation of the fungus for 23 days after the wood sample was placed on the nutrient medium. Petri dishes 2, 3, and 4 nutrient media PD, M, and YS, respectively, cultivation of the fungus for 15 days after the wood sample was placed on the nutrient medium. Recording was done by using the GelDoc XR Imaging System: $a$ - the image of the mycelium samples in the daylight; $b$ - luminescence of the mycelium samples. Recording mode: the time of accumulation $300 \mathrm{sec}$, transform function 2.

Analysis of the images obtained by using the GelDoc XR Imaging System, which monitored luminescence of the growing mycelium samples at different time points, revealed the presence of "flashing" luminescence zones with a high level of light emission (Fig. 9, Fig._9_SuppInfo.ppt).

\section{Pellet luminescence (submerged mycelial culture)}

The visible light image of the pellets grown in submerged culture on the PD medium is shown in Fig. 10. As the level of light emission by the pellets was very low, the GelDoc XR Imaging System was unable to detect their luminescence.

Glomax ${ }^{\circledR} 20 / 20$, however, detected luminescence of the pellets that had been incubated in deionized water continuously bubbled with air. Luminescence intensity of the pellets after $24 \mathrm{~h}$ incubation and the effects of external factors and the addition of different compounds are shown in Figs. 11 and 12. 

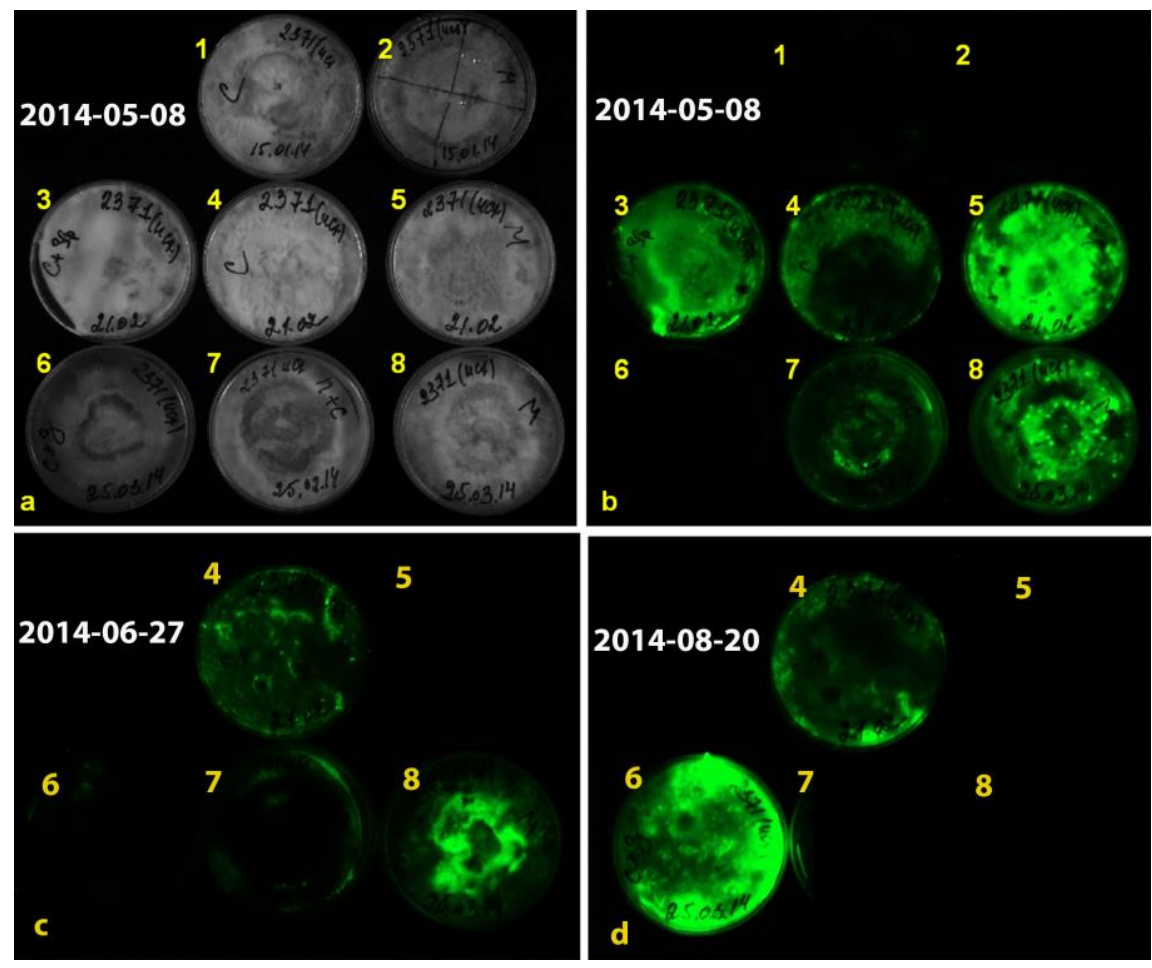

Fig. 8 - External appearance and luminescence of mycelium samples as related to the time of cultivation and the composition of the medium. 1,4 - S-agar, 2,5,8 - M-agar, 3,6 - S+G agar, 7 $\mathrm{S}+\mathrm{W}$ agar. Recording was done by using the GelDoc XR Imaging System: a - the image of the mycelial samples in the daylight; b, c, d - luminescence of the samples. Recording mode: the time of accumulation $300 \mathrm{sec}$, transform function 1.
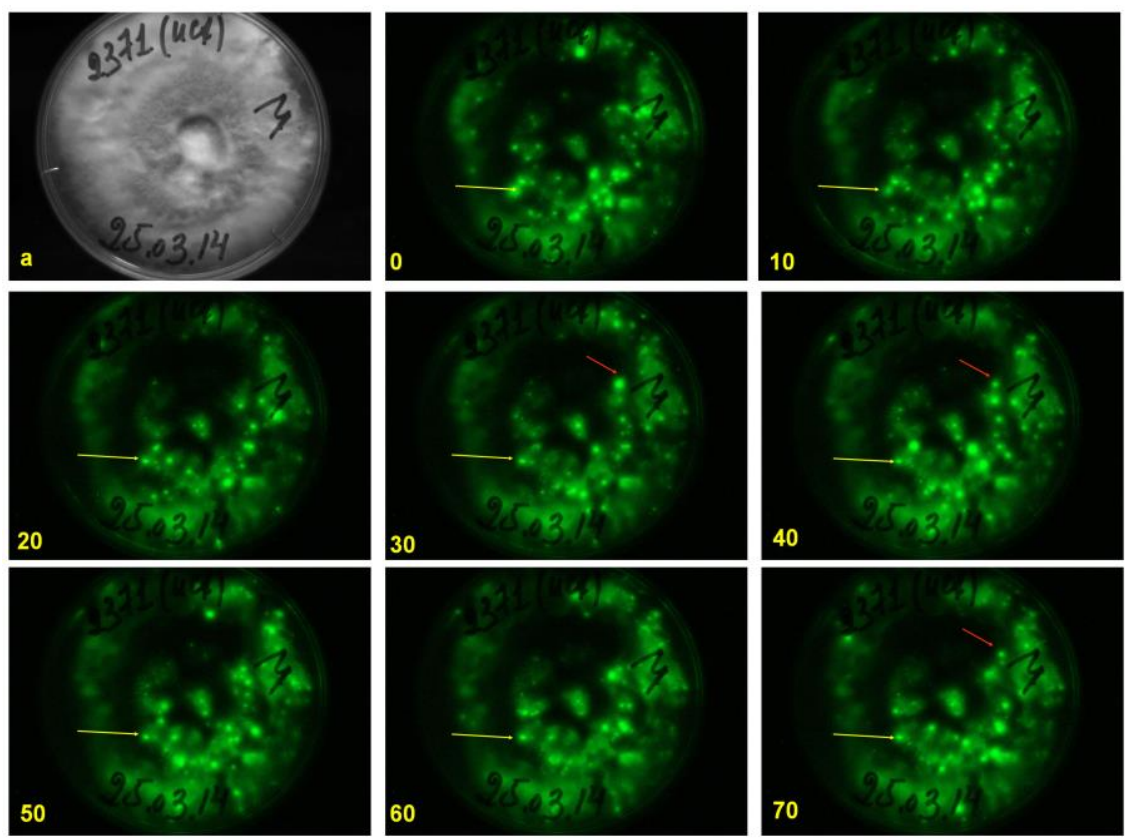

Fig. 9 - External appearance of mycelium and "flashing" luminescence zones with high levels of light emission, which were observed in the mycelium after 36 days of cultivation of the fungus. Recording was done by using the GelDoc XR Imaging System: a - external appearance of the mycelial sample in the daylight; 0 - 70 - luminescence recorded in the sample of mycelium every $10 \mathrm{~min}$. Yellow arrow - the area where the glow is recorded every 10 minutes, a red arrow - the area where the signal "appears and disappears". Recording mode: the time of accumulation $60 \mathrm{sec}$, transform function 2 . 


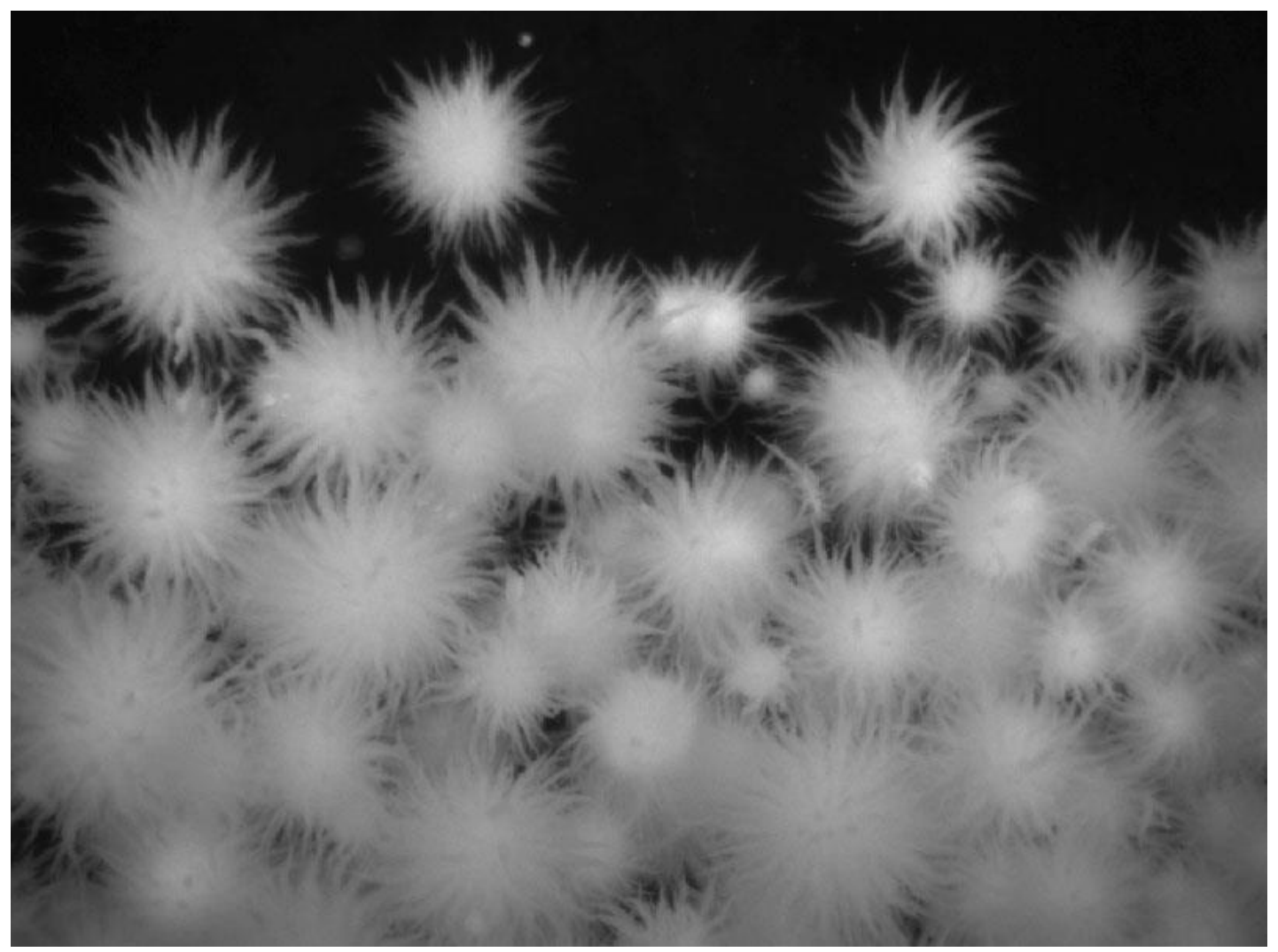

Fig. 10 - External appearance of mycelial pellets produced in the submerged culture of the fungus. Recording was done by using the GelDoc XR Imaging System in the daylight.

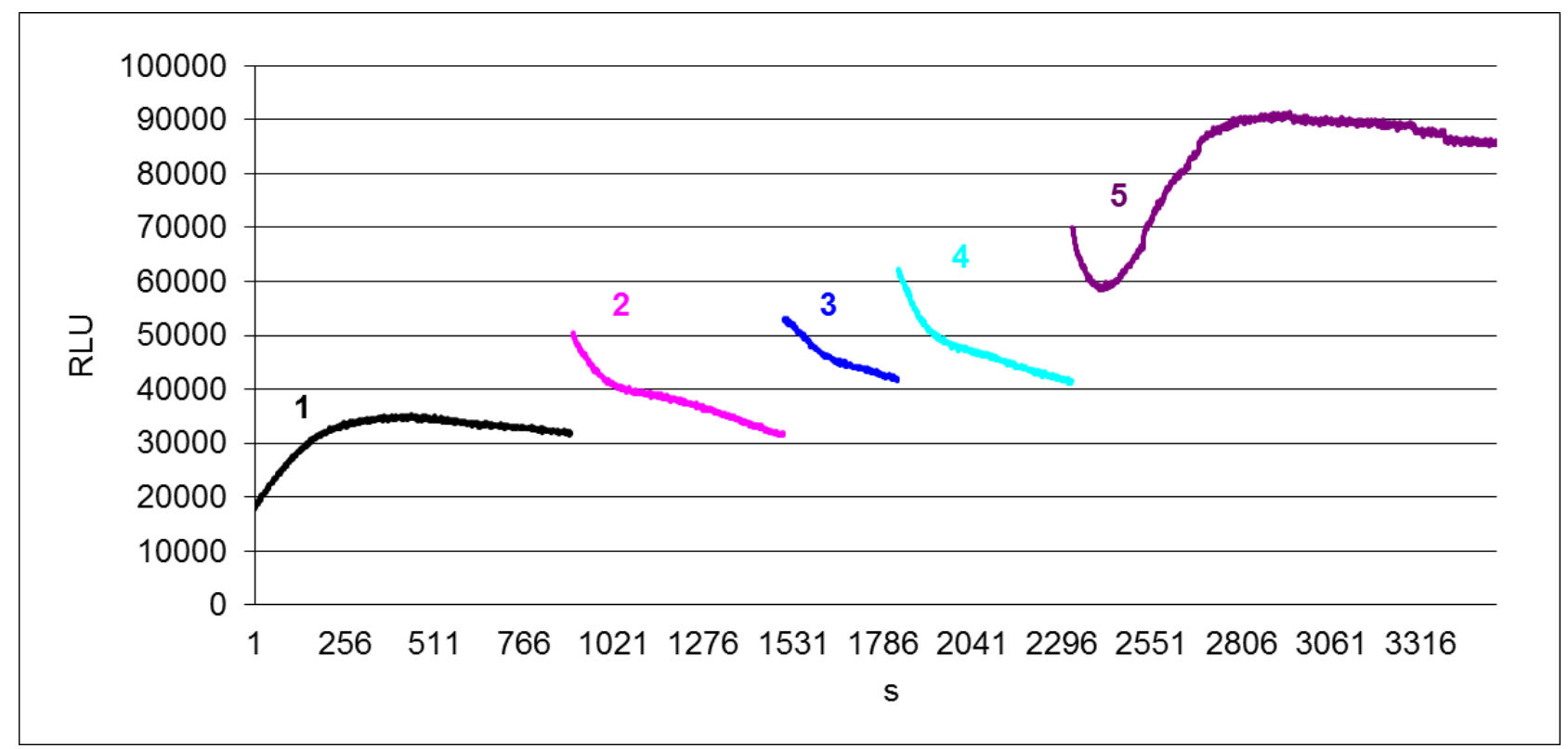

Fig. 11 - Luminescence intensity of the mycelial pellets after $24 \mathrm{~h}$ of incubation in deionized water continuously bubbled with air and changes in the luminescence level of the pellets affected by external factors and the addition of different compounds. 1 - luminescence intensity of the pellets after incubation in water; 2 - luminescence intensity after stirring of the sample; 3 - luminescence intensity after adding $5 \mu \mathrm{l}$ NADPH and mixing of the sample; 4 - luminescence intensity after adding $5 \mu \mathrm{l}$ hot extract of the fruiting bodies of nonluminous fungus Pholiota squarrosa and mixing of the sample; 5 - luminescence intensity after adding $5 \mu l$ hydrogen peroxide and mixing of the sample. Measurements were done by using Glomax ${ }^{\circledR}$ 20/20. 


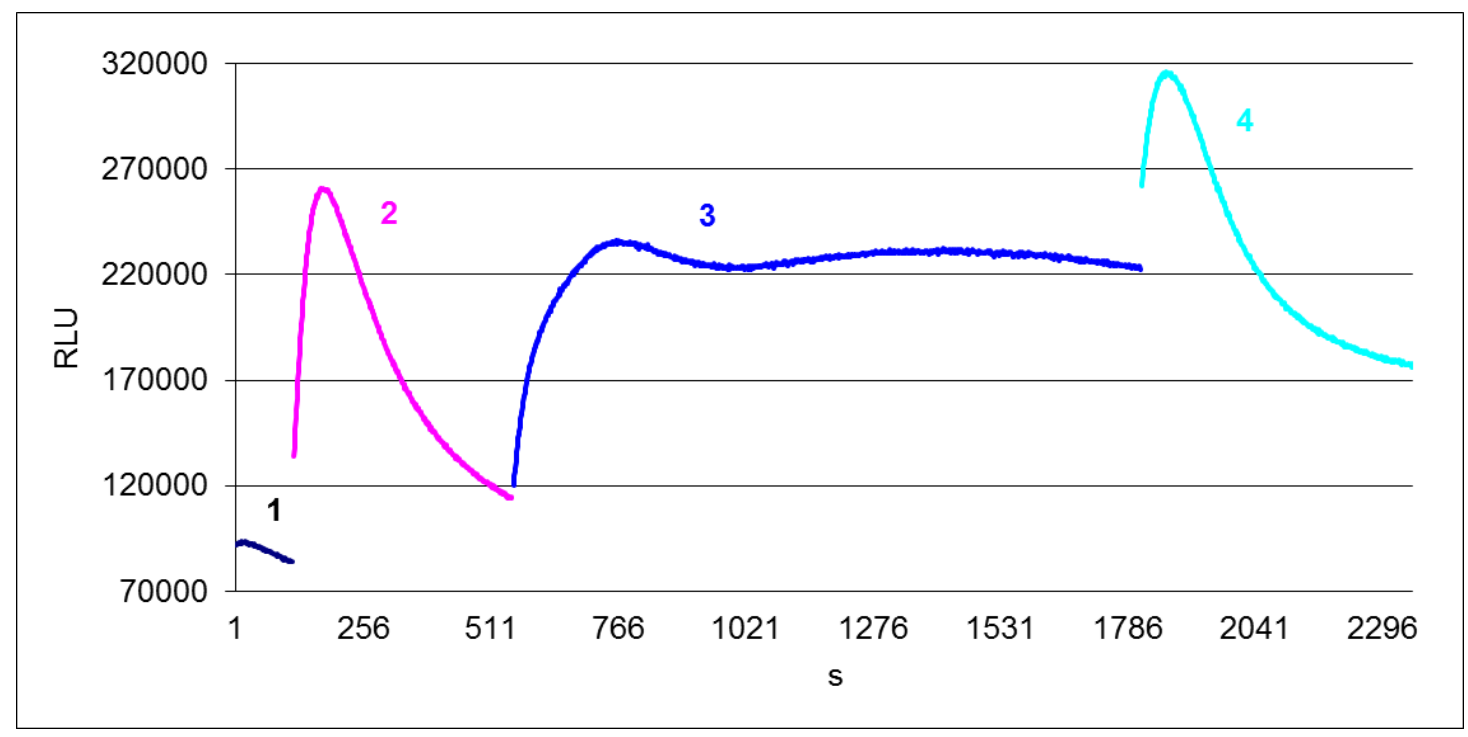

Fig. 12 - Luminescence intensity of the mycelial pellets after $24 \mathrm{~h}$ of incubation in deionized water continuously bubbled with air and changes in the luminescence level of the pellets affected by external factors and the addition of different compounds. 1 - luminescence intensity of the pellets after incubation in water; 2 - luminescence intensity after stirring of the sample; 3 - luminescence intensity after adding $5 \mu \mathrm{l}$ hydrogen peroxide and mixing of the sample; 4 - luminescence intensity after adding $5 \mu \mathrm{l}$ hot extract of the fruiting bodies of nonluminous fungus Pholiota squarrosa and mixing of the sample. Measurements were done by using Glomax ${ }^{\circledR}$ 20/20.

\section{Light emission by the luminescent system isolated from luminous mycelium}

Luminescence signals of the system isolated from the biomass of the mycelial pellets after the 6-month storage in the form of freeze-dried powder are shown in Fig. 13.

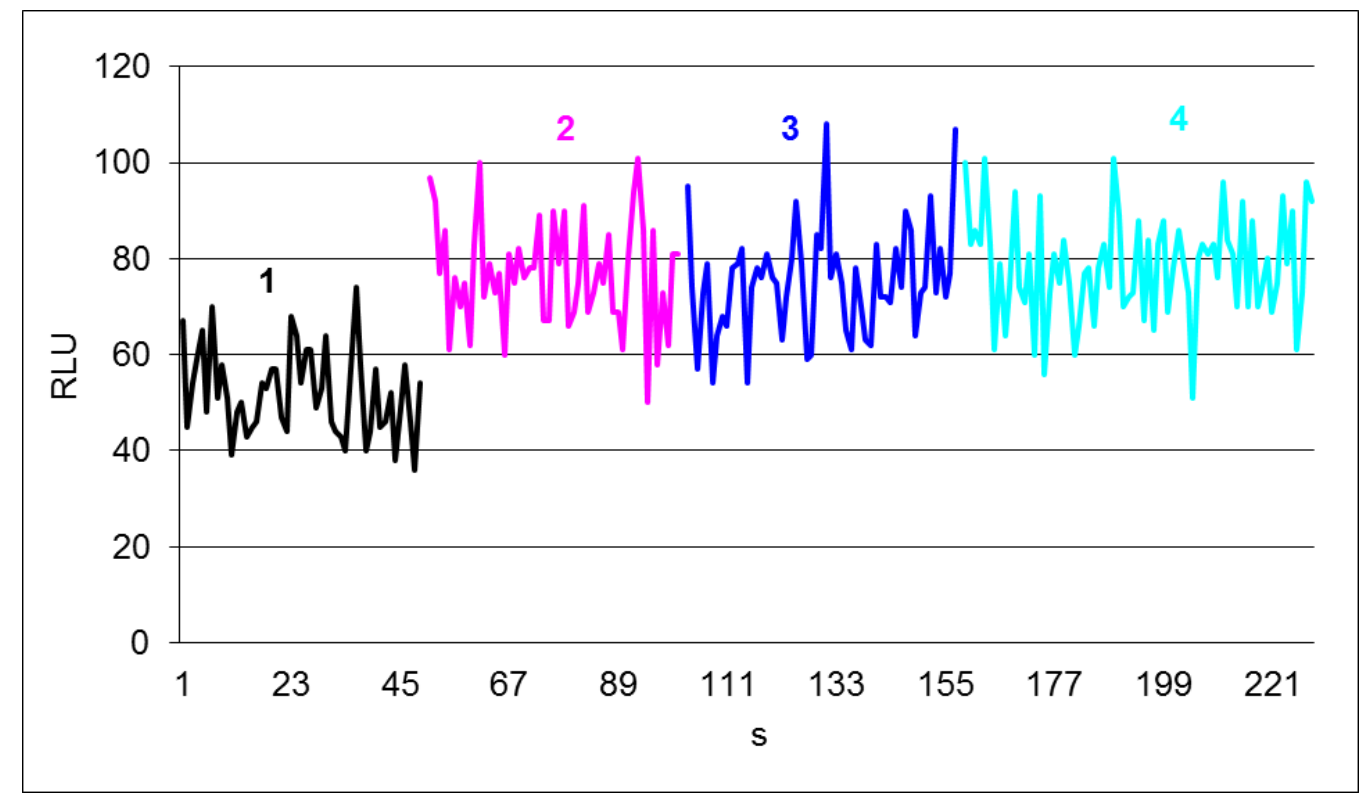

Fig. 13 - Luminescence intensity of the extract containing the luminescence system isolated from the IBSO 2371 mycelial pellets after the 6-month storage in the form of freeze-dried powder. 1 the background noise of the instrument containing an empty test-tube; 2 - luminescence intensity from the test-tube containing $100 \mu \mathrm{l}$ of the extract; 3 - luminescence intensity after adding $5 \mu 1$ NADPH to the sample; 4 - luminescence intensity after adding $5 \mu 1$ hot extract prepared by the heat treatment of the extract containing the luminescence system. Measurements were done by using Glomax ${ }^{\circledR}$ 20/20. 
The effects of different components added to the isolated luminescent system on the level of its light emission are shown in Fig. 14.

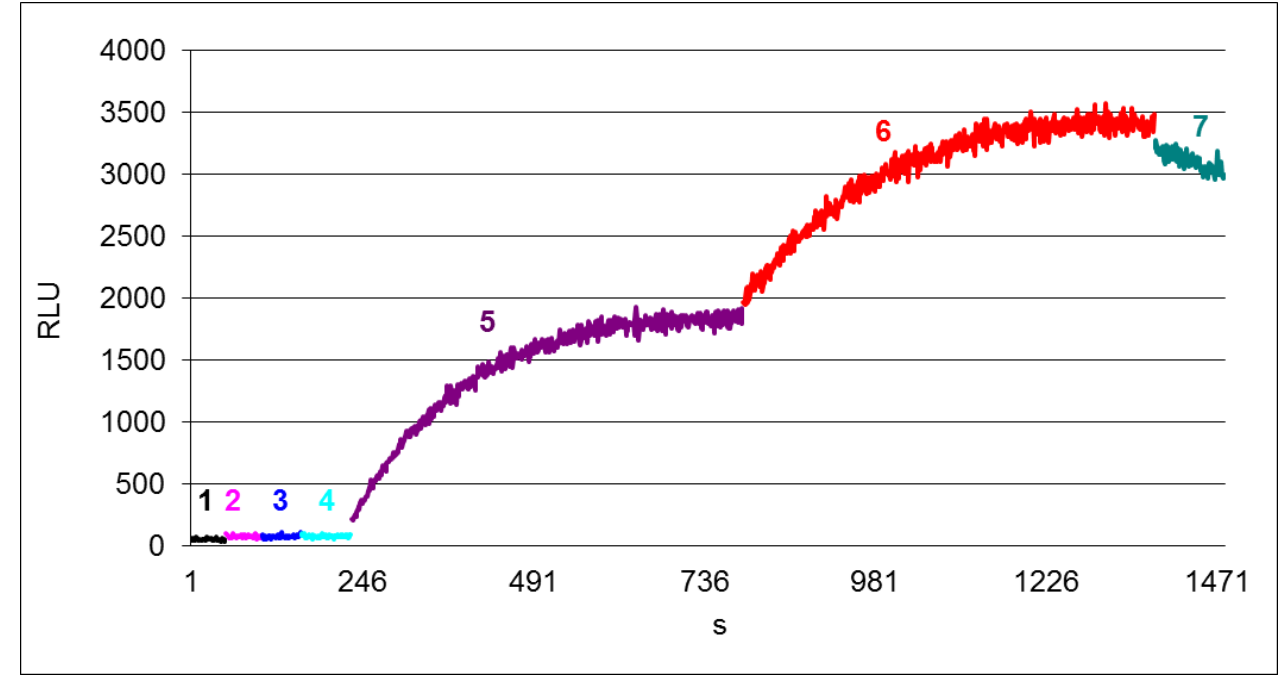

Fig. 14 - The effects of different components added to the extract of the IBSO 2371 pellet biomass that contains the luminescent system.

1 - the background noise of the instrument containing an empty test-tube;

2 - luminescence intensity from the test-tube containing $100 \mu \mathrm{l}$ of the cold extract of the IBSO

2371 mycelium;

3 - luminescence intensity after adding $5 \mu 1$ NADPH to the sample;

4 - luminescence intensity after adding $5 \mu 1$ hot extract of the IBSO 2371 mycelium;

5 - luminescence intensity after adding $5 \mu \mathrm{l}$ hot extract of luminous mycelium of the fungus

Armillaria sp. IBSO 2360;

6 - luminescence intensity after adding $5 \mu 1$ hot extract of fruiting body of the nonluminous fungus Hypholoma capnoides;

7 - luminescence intensity after adding $10 \mu 1$ hot extract of the IBSO 2371 mycelium.

The effect of hydrogen peroxide added to the luminescent system on the level of its light emission is shown in Fig. 15.

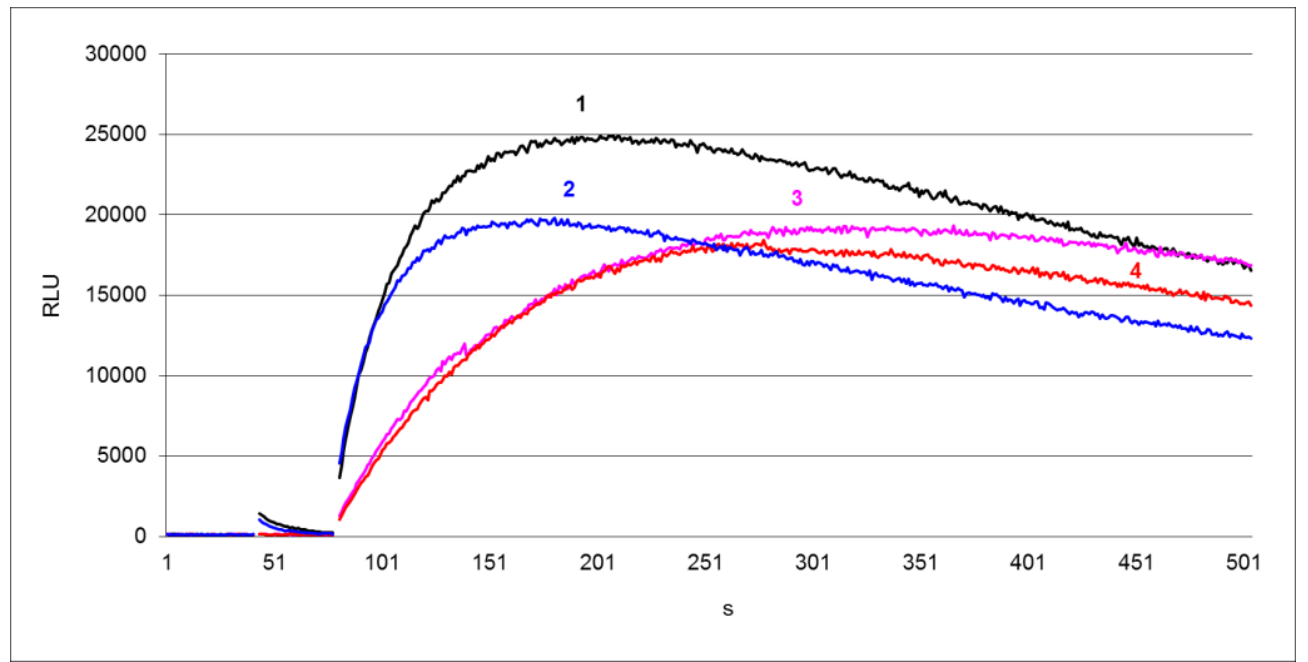

Fig. 15 - The effect of hydrogen peroxide on the luminescence level of the extract of the IBSO 2371 mycelial biomass containing the luminescence system. Experiments with sequential addition of different ingredients to the measuring microtube: 
$1-100 \mu \mathrm{l}$ extract containing the luminescence system $+5 \mu$ l hydrogen peroxide $+5 \mu$ l hot extract of the fruiting bodies of the fungus Pholiota squarrosa $+5 \mu \mathrm{NADPH}$.

$2-100 \mu \mathrm{l}$ extract containing the luminescence system $+5 \mu \mathrm{l}$ NADPH $+5 \mu$ l hydrogen peroxide + $5 \mu \mathrm{l}$ hot extract of the fruiting bodies of the fungus P.squarrosa.

$3-100 \mu 1$ extract containing the luminescence system $+5 \mu 1 \mathrm{NADPH}+5 \mu 1$ hot extract of the fruiting bodies of the fungus $P$. squarrosa.

$4-100 \mu$ l extract containing the luminescence system $+5 \mu l$ hot extract of the fruiting bodies of the fungus $P$. squarrosa $+5 \mu \mathrm{NADPH}$.

\section{Discussion}

In this study, we detected luminescent zones of wood and mycelium (isolate IBSO 2371) samples by using a Universal Hood II system. Our choice of this system was based on the following reasons. The GelDoc XR Imaging System with the Universal Hood II is intended for 1-d electrophoresis gels, dot blots, arrays and colonies. Software Quantity One ${ }^{\circledR}$ can image and analyze a wide variety of biological data, including chemiluminescent, fluorescent, radioactive, and colorstained samples. Universal Hood II is light-tight, enabling accurate detection of luminescence of the samples. The SDC camera and Software Quantity One ${ }^{\circledR}$ are sensitive enough to detect light signals of low intensities. With accumulation time between 600 and $999 \mathrm{sec}$, the diaphragm open maximally, and software enhancement, the instrument can detect even chemiluminescent signals accompanying photosynthesis. As we studied bioluminescence (light emission by living organisms visually observed in the dark), the maximum signal accumulation time in our experiments was 300 sec.

\section{Luminescent wood}

Universal Hood II is equipped with inbuilt daylight sources, enabling imaging of the sample appearance. The high-sensitivity SDC camera provides a black-and-white image of the sample in the daylight (Fig. 1a) and its own luminescence in the dark (Fig. 1b). Software Quantity One®, however, converts the black-and-white image into the color image (Fig. 1c). Comparison of images (Figs. 2 and 3) shows that the green light of the luminescent zones obtained by using the GelDoc XR Imaging System software (Fig. 2) is close to the natural color of the light emitted by them, as recorded by the Canon EOS 6D camera (Fig. 3). As shown in Fig. 3, studied samples emitted light in the green spectral region, and this is consistent with the data on basidiomycetes reported by us and other authors. Light emitting fungi are usually saprophytes or, more seldom, plant pathogens. They emit greenish light with the peak between 520 and $530 \mathrm{~nm}$ in different stages of their life cycle (Van der Burg 1943, Airth \& McElroy 1959, Wassink \& Kuwabara 1966, Lavelle et al. 1972, O'Kane et al. 1990, Shimomura 2006, Desjardin et al. 2008, Bondar et al. 2011, 2012).

The wood samples that we collected 22 months ago (as of the time of writing) still retain their luminescence, as detected by the GelDoc XR Imaging System. This is in good agreement with the data of other authors, suggesting that wood remains luminescent for long periods of time (Deheyn \& Latz 2007, Sabharwal et al. 1983, Bose 1930). These data are indicative of high viability of the mycelium that we study and suggest several conclusions. Judging from our experience, luminescent wood found in the forest does not need to be delivered to the laboratory immediately and aseptically. However, it is important to keep the samples moist. Our observations showed that as the wood dried, the intensity of its luminescence dropped to the level undetected by the GelDoc XR Imaging System and Glomax ${ }^{\circledR}$ 20/20. Moistening of the dried samples did not restore their luminescence but caused lower fungi to grow on the wood surface.

Thus, our studies confirm that the main condition for long-lasting luminescence of wood is high humidity. At high humidity levels, not only does the substrate mycelium remain viable but also luminescent aerial mycelium develops on the wood (Figs. 2 - 4).

Although the samples were not maintained under aseptic conditions, no lower fungi or bacteria were observed to grow on the wood surface, suggesting an assumption that luminescent mycelium has bacteriostatic properties, inhibiting development of microflora. However, we failed 
to prepare pure culture by using luminous aerial mycelium that had grown on the surface of the wood stored under laboratory conditions. When solid nutrient media were seeded with mycelial inoculum and the fungus was grown in culture, only colonies of bacteria and lower fungi developed on the agar surface. A possible reason for this may be the difference between the compositions of nutrients in the laboratory media and in the natural substrate of the tropical forest. Wood is a nutrient-poor substrate, and not all organisms can grow on it. Therefore, if the mycelium that we used in our study was able to colonize the natural wood substrate in the tropical forest, then, during its subsequent development, it managed to inhibit the growth of concomitant microflora. On the nutrient-rich medium, concomitant microflora (lower fungi and bacteria), whose growth rate is higher, might have an advantage over the higher fungus, suppressing its growth and, thus, preventing us from preparing pure culture of luminescent mycelium. Thus, we assume that the luminescent mycelium used in our study releases metabolites that have a bacteriostatic effect and are capable, under certain conditions, of inhibiting the growth of concomitant microflora. Over 22 months of observation, we have not detected any changes in the size, shape, or color of luminous wood samples. Hence, we suppose that the natural substrate contains sufficient amounts of nutrients to support viability and luminescence of the fungus over an extended time period (years) when maintained at certain temperature and humidity.

Our study showed that pure mycelial culture of this fungus could be prepared by using inner layers of wood as inoculum: these layers are threaded with luminescent mycelium but do not contain microorganisms and their spores, which are present on the wood surface. The use of the GelDoc XR Imaging System considerably facilitates microbiological procedures of deriving the culture of fungal mycelium from the wood. Analysis of the images points to the zones with the maximum luminescence (Fig. 5). We assume that high luminescence intensity may be determined by the large number of fungus hyphae per unit area of the natural wood substrate and a high biotic potential of the growing mycelium (its metabolic rate). The use of this inoculum increases the probability of successful cultivation of mycelium on nutrient media.

Based on imaging of luminescent wood samples placed on the agar medium, we selected Petri dishes with high levels of light emission by the inoculum (Fig. 6b) and monitored the growth of colonies of mycelium and development of its luminescence on different nutrient media (Fig. 7). The detection of the luminescence signal from mycelium was the proof of the growth of the luminous fungus, excluding the possibility of accidental contamination. By the subsequent passage of the luminescent mycelium, we managed to prepare a stable culture of the fungus - isolate IBSO 2371.

\section{Properties of mycelial culture on solid nutrient media}

Cultivation of mycelium on solid nutrient media of different compositions (Figs. 7 and 8) revealed differences in mycelium growth rates and luminescence intensities. Mycelium grew faster and showed higher intensity of light emission when cultivated on the M agar and YM agar, which contained malt extract, and on the S agar. On the PD agar, the growth rate of mycelium was slower, and its luminescence intensity was lower than when it was grown on the $\mathrm{M}$ agar. The slowest growth of mycelium was observed on the YS agar, which contained yeast extract and cane sugar. Fig. 8 shows mycelium at different time points during cultivation in Petri dishes on different media. Recording of luminescence of these samples using Glomax ${ }^{\circledR}$ 20/20 suggests that bright luminescence (more than $5 \times 10^{6} \mathrm{RLU} / \mathrm{sec}$ ) that can be seen in the dark was first detected between days 20 and 40 of mycelial growth. The peak of mycelium luminescence (up to $2.9 \times 10^{8} \mathrm{RLU} / \mathrm{sec}$ ) was recorded between days 80 and 100; then it declined gradually, remaining rather high (up to 4$5 \times 10^{6} \mathrm{RLU} / \mathrm{sec}$ ) for a long time (140 days or longer). Changes in luminescence intensity of mycelium cultivated on the $\mathrm{M}$ agar and $\mathrm{S}$ agar media occurred at different time points. Mycelium grown on the $\mathrm{M}$ agar showed the luminescence level of $10^{6} \mathrm{RLU} / \mathrm{sec} 2-5$ days earlier than the mycelium grown on the $\mathrm{S}$ agar. The peak of luminescence of mycelium on the M agar was also observed at earlier time points (between days 49 and 100 of the culture) compared with the peak of luminescence of mycelium on the S agar (between days 85 and 104 of the culture). Luminescence 
decay occurred earlier on the $\mathrm{M}$ agar than on the S agar, too: at days 102 and 113, respectively. A supplementary study showed that when water was replaced by the extract of the coconut palm wood (S agar + wood ext.), bright luminescence of mycelium grown on this medium lasted 150 days, with the peak observed between days 100 and 120 . We also found that the use of wheat grain extract instead of water in the $\mathrm{S}$ agar ( $\mathrm{S}$ agar + grain wheat ext.) prolonged the light emission by mycelium considerably (to 170 days) and increased the time period of the maximum bright

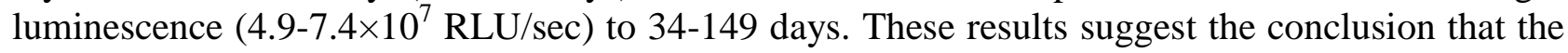
duration and intensity of luminescence of the mycelium used in this study were considerably influenced by the composition of the nutrient medium on which it was grown.

We observed the following distinctive characteristics of the culture of this mycelium on solid nutrient media compared to other cultures of luminous fungi that we had studied (Medvedeva et al. 2014): (i) the presence of local zones showing high luminescence levels, which change luminescence intensity over a short time (Fig. 9, Fig._9_SuppInfo.ppt) and, thus, seem to emerge, travel, and disappear; (ii) the low growth rate of mycelium - one of the lowest growth rates among our cultures of luminescent fungi (Panellus stipticus, Mycena citricolor, Omphalotus japonicus, Neonothopanus nambi, Armillaria sp. - 37 isolates); (iii) long-lasting luminescence of mycelium: 150 days or longer, with light emission intensity reaching $4-5 \times 10^{6} \mathrm{RLU} / \mathrm{sec}$. Moreover, the maximum luminescence intensity of this mycelium can reach $2.9-5 \times 10^{8} \mathrm{RLU} / \mathrm{sec}$, and this is comparable with the luminescence intensity of mycelium of known basidiomycetes (Medvedeva et al. 2014).

\section{Properties of mycelial culture in liquid nutrient medium}

Mycelium pellets produced by growing the IBSO 2371 culture in the liquid nutrient medium did not generate a luminescent signal; even Glomax ${ }^{\circledR} 20 / 20$ did not detect one. The recorded signal intensity, 60 - 100 RLU/sec (Fig. 10), was not discernible against the background noise of the instrument. A similar case is described in the literature, when mycelium of Panellus stipticus emitted light visible in the dark when grown on solid nutrient media but lost this ability when grown on/in the liquid nutrient medium (Shimomura 2006).

Luminescence intensity of mycelium, however, can be considerably enhanced (several-fold or even by orders of magnitude) by rinsing it in water to remove the nutrient medium and metabolites (Mori et al. 2011, Bondar et al. 2012, 2013). The use of this technique was successful in our study of IBSO 2371 mycelium. After the pellets had been incubated in deionized water for 24 h, a luminescent signal was detected by the Glomax® 20/20 luminometer (Figs. 11 and 12).

We revealed individual differences in the levels of initial light emission by pellets and changes of luminescence caused by different factors (mechanical damage during stirring of the sample and addition of solutions of various substances). As shown in Figs. 11 and 12, luminescence intensities of pellets may differ considerably. For example, the initial luminescence signal (Fig. 11) of one of the pellets was $1.8 \times 10^{4} \mathrm{RLU} / \mathrm{sec}$, and it increased with time. The initial luminescence level of another pellet (Fig. 12), however, was $1 \times 10^{6} \mathrm{RLU} / \mathrm{sec}$, and it insignificantly decreased with time. After the sample was stirred, changes in the light emission by the pellets were different, too. The reasons for enhancement of the luminescence signal during stirring may be both mechanical damage and an increase in oxygen concentration in the medium containing the sample. This response is characteristic of luminescent bacteria (Gitelson et al. 1984) and mycelium of N. nambi (Bondar et al. 2013). In spite of considerable individual differences in luminescence intensity of pellets mentioned above, there are common trends in the changes of their luminescence in response to the impact of an external factor (or several factors). For instance, the level of luminescence of the pellets increased after addition of $\mathrm{H}_{2} \mathrm{O}_{2}$, and that increased level was retained for a long time; no substantial changes were observed in the luminescence signal upon the addition of NADPH. All pellets increased light emission almost immediately upon impact of an external factor.

\section{Properties of the luminescence system isolated from mycelial biomass}

As mentioned above, the signal intensity of 60 - 100 RLU corresponds to the background 
noise of the Glomax ${ }^{\circledR}$ 20/20 luminometer. Thus, the data presented in Fig. 13 suggest that the luminescence system isolated from the pellet biomass had no ability to emit light. The addition of NADPH, which is a trigger of the luminescent reaction (Oliveira et al. 2012), and the hot extract of the pellets, which could contain the luminescent reaction substrate, did not induce luminescence the signal detectable by Glomax ${ }^{\circledR} 20 / 20$ did not exceed the background noise level. The absence of luminescence could be due to one of the following reasons: 1 - degradation of the luminescence system enzymes during isolation of this system out of mycelial biomass; 2 - the absence of the luminescent reaction substrate, with the luminescence system enzymes remaining active; 3 degradation of the enzyme system and the absence of the luminescent reaction substrate.

Our study showed (Fig. 14) that sequential addition of NADPH and hot extracts of the luminescent mycelium of Armillaria sp. (IBSO 2360) or nonluminous fruiting bodies of Hypholoma capnoides to the luminescence system isolated from the biomass of the IBSO 2371 pellets initiated generation of the luminescence signal detectable by Glomax ${ }^{\circledR}$ 20/20. A similar effect was observed when hot extracts of the fruiting bodies of other nonluminous fungi were added: Pholiota squarrosa (Fig. 15), Cortinarius sp. and Chroogomphus rutilus (data are not presented here). Thus, enzymes in the isolated luminescence system retained their activity, and the factor that limited luminescence was the absence (or very low amounts) of the reaction substrate and NADPH. Recently it was reported that hot extracts the fruiting bodies of nonluminous fungi Pholiota squarrosa, Tricholoma sp., Phellinus sp., Russula foetens, Clitocybe sp. increase the luminescence of in vitro N. nambi system (Supporting Information to the article Purtov et al. 2015).

Comparison of the data obtained (Figs. 11, 12 and 15) shows that $\mathrm{H}_{2} \mathrm{O}_{2}$ produced different effects on the luminescence of pellets and on the luminescence system isolated out of them. The addition of $\mathrm{H}_{2} \mathrm{O}_{2}$ to the luminescence system (Fig. 15) changed the kinetics of the luminescence signal without increasing its intensity, while in the case of pellets (Figs. 11 and 12), hydrogen peroxide increased the intensity of luminescence and changed its kinetics.

Previously, Oliveira et al. (2012) reported: "All cross-reactions with extracts from luminescent species yielded positive results, independent of lineage, whereas no light was emitted in cross-reactions with extracts from non-luminescent species. These results support the hypothesis that all lineages of luminescent fungi share the same type of luciferin and luciferase, that there is a single luminescent mechanism in the fungi and that fungal luciferin is not a ubiquitous molecule in fungal metabolism". The enhancement of the luminescence signal caused by addition of the hot extract of Armillaria sp. (IBSO 2360) luminescent biomass, which contained the luminescent reaction substrate, to the luminescence system of IBSO 2371 is consistent with this hypothesis. At the same time, the increase in the luminescence caused by the addition of the hot extracts of nonluminous fungal biomass contradicts this hypothesis. Our results suggest that there are nonluminous fungi that synthesize a substance (substances) enhancing light emission of the luminescence system. Moreover, this effect is not solely a characteristic of the luminescence system of IBSO 2371. We observed initiation of luminescence by hot extracts of nonluminous fungi in our studies of luminescence systems isolated out of mycelia of such luminous fungi as $N$. nambi and some of Armillaria species (unpublished data). It is not clear yet whether the extracts of these fungi contain the luminescent reaction substrate or a component (components) stimulating luminescence. Further research is needed to study these issues. The data obtained in this study point to a number of differences between IBSO 2371 and cultures of other luminous fungi maintained in the Collection of IBP SB RAS. Now that we have pure mycelium culture, we may be able to perform taxonomic identification of the fungus by using phylogenetic analysis.

\section{Acknowledgements}

The authors are grateful to Prof. A. Frank, Director of North Borneo Biostation, for the opportunity to carry out studies of glowing wood; to Nadezhda N. Kudashova, a senior researcher at the Institute of Biology and Biophysics at the Tomsk University, for identifying the species of nonluminous fungi. This study was supported by grant no. 11.G34.31.0058 (RF Government) and Projects no. 71 (SB RAS). 


\section{References}

Airth RL, McElroy WD. 1959 - Light emission from extract of luminous fungi. J. Bacteriol. 77, 249-250.

Bondar VS, Puzyr AP, Purtov KV, Medvedeva SE, Rodicheva EK, Gitelson JI. 2011 - The luminescent system of the luminous fungus Neonothopanus nambi. Doklady Biochemistry and Biophysics. 438, 138-140.

Bondar VS, Rodicheva EK, Medvedeva SE, Tyulkova NA, Tyaglik AB, Shpak BA, Gitelson JI. 2013 - On the mechanism of luminescence of the fungus Neonothopanus nambi. Doklady Biochemistry and Biophysics. 449, 80-83.

Bondar VS, Shimomura O, Gitelson JI. 2012 - Luminescence of higher mushrooms. Journal of Siberian Federal University. Biology. 5(4), 331-351.

Bose SR. 1930 - Relation of sunlight to the light of luminous wood. Die Naturwissenschaften. 18(36), 787.

Brandl H. 2011 - Luminescent wood in coal and ore mines. Fungi. 4(2), 5-9.

Chew ALC, Desjardin DE, Tan Y-S, Musa MdY, Sabaratnam V. 2015 - Bioluminescent fungi from Peninsular Malaysia - a taxonomic and phylogenetic overview. Fungal Diversity. 70, 149--187.

Deheyn DD, Latz MI. 2007 - Bioluminescence characteristics of a tropical terrestrial fungus (Basidiomycetes). Luminescence. 22, 462-467.

Desjardin DE, Oliveira AG, Stevani CV. 2008 - Fungi bioluminescence revisited. Photochemical \& Photobiological Sciences. 7, 170-182.

Gitelson JI, Rodicheva EK, Medvedeva SE, Primakova GA, Bartsev SI, Kratasyuk GA, Petushkov VN, Mezhevikin VV, Vysotski ES, Zavoruev VV, Kratasyuk VA. 1984 - Luminous Bacteria. Novosibirsk. Nauka. (In Russian).

Harvey EN. 1952 - Bioluminescence. New York: Academic Press.

Harvey EN. 1957 - A history of luminescence from the earliest times until 1900. Baltimore, Maryland: J.H. Furst Company.

Jaczewski AA. 1933 - Elements de la mycologie. Oeuvre posthume, redigee par N. Naoumoff. Edition de l'etat section de l'agriculture (colchos et sovshos). Moscov - Leningrad. Pp. 579580. (In Russian).

Lavelle MMF, Durosay P, Michelson AM. 1972. - Bioluminescence. Luminescence des champignons lumineux. Comptes Rendus de l'Académie des Sciences. Paris. Série D. 275, 1227-1230.

Medvedeva SE, Artemenko KS, Krivosheenko AA, Rusinova AG, Rodicheva EK, Puzyr AP, Bondar VS. 2014 - Growth and light emission of luminous basidiomycetes cultivated on solid media and in submerged culture. Mycosphere. 5, 565-577.

Mori K, Kojima S, Maki S, Hirano T, Niwa H. 2011 - Bioluminescence characteristics of the fruiting body of Mycena chlorophos. Luminescence. 26, 604--610.

O'Kane DJ, Lingle WL, Porter D, Wampler JE. 1990 - Spectral analysis of the bioluminescence of Panellus stipticus. Mycologia. 82, 607-616.

Oliveira AG, Desjardin DE, Perry BA, Stevani CV. 2012 - Evidence that a single bioluminescent system is shared by all known bioluminescent fungal lineages. Photochemical \& Photobiological Sciences. 11, 848-852.

Purtov KV, Petushkov VN, Baranov MS, Mineev KS, Rodionova NS, Kaskova ZM, Tsarkova AS, Petunin AI, Bondar VS, Rodicheva EK, Medvedeva SE, Oba Y, Oba Y, Arseniev AS, Lukyanov S, Gitelson JI, Yampolsky IV. 2015 - The Chemical Basis of Fungal Bioluminescence. Angewandte Chemie International Edition. 54(28), 8124-8128.

Sabharwal SC, Kathuria SP, Dhingra MM. 1983 - A new bioluminescent fungal system. Journal of Biosciences. 5(1), 53-62.

Shimomura O. 2006 - Bioluminescence: chemical principles and methods. Singapore: World Scientifc Publishing Co. Pte. Ltd. Pp. 266-300. 
Van der Burg A. 1943 - Spektrale onderzoekingen over chemo- en bioluminescentie. Utrecht: University of Utrecht.

Wassink, EC, Kuwabara S. 1966 - Bioluminescence in Progress. Princeton Univ. Press. New Jersey. P. 247. 